\title{
Assessment of the impact of mitochondrial genotype upon drug-induced mitochondrial dysfunction in platelets derived from healthy volunteers
}

\author{
Amy L. Ball ${ }^{1} \cdot$ Katarzyna M. Bloch² $\cdot$ Lucille Rainbow $^{3} \cdot$ Xuan Liư $^{3} \cdot$ John Kenny $^{3} \cdot$ Jonathan J. Lyon ${ }^{4}$. \\ Richard Gregory $^{3} \cdot$ Ana Alfirevic $^{2} \cdot$ Amy E. Chadwick $^{1}$ (i)
}

Received: 9 July 2020 / Accepted: 21 January 2021 / Published online: 13 February 2021

(c) The Author(s) 2021

\begin{abstract}
Mitochondrial DNA (mtDNA) is highly polymorphic and encodes 13 proteins which are critical to the production of ATP via oxidative phosphorylation. As mtDNA is maternally inherited and undergoes negligible recombination, acquired mutations have subdivided the human population into several discrete haplogroups. Mitochondrial haplogroup has been found to significantly alter mitochondrial function and impact susceptibility to adverse drug reactions. Despite these findings, there are currently limited models to assess the effect of mtDNA variation upon susceptibility to adverse drug reactions. Platelets offer a potential personalised model of this variation, as their anucleate nature offers a source of mtDNA without interference from the nuclear genome. This study, therefore, aimed to determine the effect of mtDNA variation upon mitochondrial function and drug-induced mitochondrial dysfunction in a platelet model. The mtDNA haplogroup of 383 healthy volunteers was determined using next-generation mtDNA sequencing (Illumina MiSeq). Subsequently, 30 of these volunteers from mitochondrial haplogroups $H, J, T$ and $U$ were recalled to donate fresh, whole blood from which platelets were isolated. Platelet mitochondrial function was tested at basal state and upon treatment with compounds associated with both mitochondrial dysfunction and adverse drug reactions, flutamide, 2-hydroxyflutamide and tolcapone (10-250 $\mu \mathrm{M})$ using extracellular flux analysis. This study has demonstrated that freshly-isolated platelets are a practical, primary cell model, which is amenable to the study of drug-induced mitochondrial dysfunction. Specifically, platelets from donors of haplogroup $J$ have been found to have increased susceptibility to the inhibition of complex I-driven respiration by 2-hydroxyflutamide. At a time when individual susceptibility to adverse drug reactions is not fully understood, this study provides evidence that inter-individual variation in mitochondrial genotype could be a factor in determining sensitivity to mitochondrial toxicants associated with costly adverse drug reactions.
\end{abstract}

Keywords 2-Hydroxyflutamide $\cdot$ Adverse drug reaction · Flutamide $\cdot$ Haplogroup $\cdot$ Mitochondria $\cdot$ mtDNA $\cdot$ Platelets · Tolcapone

Amy L. Ball and Katarzyna M. Bloch are joint first authors.

Ana Alfirevic and Amy E. Chadwick are joint last authors.

Amy E. Chadwick

Amy.Chadwick@liverpool.ac.uk

1 Department of Pharmacology and Therapeutics, MRC Centre for Drug Safety Science, University of Liverpool, Liverpool, UK

2 The Wolfson Centre for Personalised Medicine, Department of Pharmacology and Therapeutics, University of Liverpool, Liverpool, UK
3 Centre for Genomic Research, Institute of Integrative Biology, University of Liverpool, Liverpool, UK

4 GSK GlaxoSmithKline, Safety Assessment, Ware, UK 


\section{Introduction}

Drug-induced mitochondrial dysfunction is a major pathway associated with adverse drug reactions which has led to the withdrawal of therapeutic compounds from the market (Massart et al. 2018). Nonetheless, many of the compounds that induce mitochondrial dysfunction can be administered to the vast majority of patients without reaction, indicating the presence of patient-specific risk factors (Chu et al. 1998; Olanow and Watkins 2007).

The mitochondrial genome is a 16,569 base-pair circular DNA encoding 22 tRNAs and 2 rRNAs as well as 13 subunits of the electron transport chain (ETC) respiratory complexes and ATP synthase, together constituting the necessary machinery for oxidative phosphorylation. In contrast to the nuclear genome, the mitochondrial genome is maternally inherited, resulting in negligible recombination; thus dividing the population into discrete haplogroups characterised by a set of single nucleotide polymorphisms (SNPs) (Taylor and Turnbull 2005).

Several studies have indicated that the mitochondrial genome may be a factor in the onset of adverse drug reactions, including those associated with linezolid and streptomycin (Chen et al. 2007; Pacheu-Grau et al. 2012; Penman et al. 2020). In addition, drug-induced mitochondrial dysfunction is an important part of preclinical screening in the pharmaceutical industry. However, research to elucidate the triad of the mitochondrial genome, drug-induced mitochondrial dysfunction and adverse drug reactions is limited. This is due, in-part, to current screening tools which neglect the inter-individual genetic diversity present at the population level (Will and Dykens 2014). Thus, research is required to define the potential importance of mitochondrial genetics in defining individual susceptibility to adverse drug reactions, as well as to provide the practical models required for the field to advance. Therefore, the aim of this study was to evaluate differences in drug-induced mitochondrial dysfunction between mitochondrial haplogroups using freshly isolated human platelets; isolated from healthy volunteers of known haplogroup. Platelets were selected due to their accessibility via a minimally invasive technique (venepuncture) and their abundance of mitochondria and mitochondrial DNA (mtDNA). The anucleate nature of platelets also enables the effect of mtDNA variation to be determined without interference from the nuclear genome (Thon and Italiano 2012).

The present study examined the mitochondrial genome of a cohort of 383 healthy volunteers from within an existing DNA biobank using whole genome sequencing to determine mitochondrial haplogroup. Following haplogroup assessment, volunteers from mitochondrial haplogroups $H, J, T$ and $U$ (the four most common haplogroups in the cohort and in England) were recalled to donate fresh whole blood from which platelets were isolated (Eupedia 2016). Basal bioenergetic function was assessed using extracellular flux (XF) analysis to quantify key parameters of mitochondrial respiratory function and individual respiratory complexdriven respiration.

The effect of haplogroup upon susceptibility to druginduced mitochondrial dysfunction was then examined using three therapeutic compounds that are known to induce mitochondrial dysfunction via two classic modes of direct ETC damage: flutamide and 2-hydroxyflutamide (primary metabolite of flutamide) via respiratory complex inhibition, and tolcapone via an uncoupling mechanism. All three compounds are associated with adverse drug reactions and have either been withdrawn or received a boxed warning as a result (Chu et al. 1998; Olanow and Watkins 2007; Ball et al. 2016; Longo et al. 2016; Grünig et al. 2017). Compared with its parent compound, 2-hydroxyflutamide has a higher $\mathrm{C}_{\max }(4.4 \mu \mathrm{M}$ vs. $72.2 \mathrm{nM})$ and is an inhibitor of respiratory complex II as well as respiratory complex I; therefore, the metabolite was selected to investigate the association of haplogroup upon susceptibility to perturbations at specific respiratory complexes (Ball et al. 2016). Finally, the results from basal and drug-induced mitochondrial (dys)function were pooled from platelets of all haplogroups to assess the utility of measuring basal mitochondrial function in platelets as a means to predict susceptibility to drug-induced mitochondrial dysfunction.

\section{Materials and methods}

\section{Materials}

All DNA library preparation reagents were purchased from Fluidigm (CA, USA) unless otherwise stated. All sequencing reagents were purchased from Illumina (CA,USA) unless otherwise stated. All XF assay consumables were purchased from Agilent Technologies (CA, USA) unless otherwise stated. All other reagents and chemicals were purchased from Sigma Aldrich (Dorset, UK) unless otherwise stated.

\section{Cohort}

This study was approved by the North West of England Research Ethics Committee and all participants gave written informed consent. Study eligibility and exclusion criteria have been published previously (Alfirevic et al. 2012).

Samples were selected from a study by Faulkner et al. in which 600 healthy volunteers were recruited from the North West of England and $100 \mathrm{~mL}$ of blood was collected for DNA extraction (Faulkner et al. 2016). The most recently collected 384 DNA samples were selected for mitochondrial genotyping to maximise volunteer recall. Volunteer 
confidentiality was maintained at all times by double coding DNA samples and by restricting access to participant personal data to trained clinical personnel.

Volunteers were $64 \%$ female and $36 \%$ male with a mean age of 29 years (range 18-60 years). The ethnicity of the volunteers also reflected regional diversity; primarily Caucasian (white north European ancestry; 84\%) with Asian Indian (6\%), Chinese (4\%) and Black (1\%) as minority populations.

\section{DNA isolation and library preparation}

Multiplex amplicon tagging using the Fluidigm 48.48 Access Array Integrated Fluidics Circuit (IFC) System was performed on 384 DNA samples according to the manufacturer's protocol using primers designed for the mitochondrial genome (Fluidigm Assay Design Report 5758_AAP_15.D1; cycling conditions detailed in Supplementary Fig. 1). Sample input was $100 \mathrm{ng}$ and all harvested PCR products were amplified and tagged with a unique barcode (1-384; conditions for incorporation of Illumina barcodes are detailed in Supplementary Fig. 2).

All barcoded, amplified PCR products generated on the 48.48 Access Array IFC underwent quality control using the Fragment Analyser and concentrations were measured by a Qubit HS kit using a 96-well plate reader. The PCR products were then pooled to create four PCR product libraries using the Mosquito X1 (TTP labtech, Melbourn, England). Following pooling, the libraries were cleaned using Ampure XP beads (1:1). The quantity and quality of each pool was assessed by the Bioanalyser (Agilent 2000) and subsequently by qPCR using the Illumina Library Quantification Kit (Kapa Biosystems, UK) on a Light Cycler LC480II (Roche, Switzerland) according to the manufacturer's instructions. The four PCR product libraries were pooled in an equimolar ratio prior to sequencing.

\section{MtDNA sequencing}

Template DNA (from PCR product libraries) was denatured according to the protocol described in the Illumina cBot user guide. Briefly, $5 \mu \mathrm{L}$ of non-denatured library was added to $5 \mu \mathrm{L} 0.1 \mathrm{M} \mathrm{NaOH}$ and $5 \mu \mathrm{L} 200 \mathrm{mM}$ Tris- $\mathrm{HCl} \mathrm{pH}$ 8.0. The library mix $(10 \mathrm{pM})$ and Read1, Read 2 and Read3 sequencing primers (diluted with hybridisation buffer) were then added to the MiSeq reagent cartridge, pre-loaded with clustering and sequencing reagents. The $2 \times 150$ bp pairedend sequencing was performed on one lane of an Illumina MiSeq with v2 chemistry. During sequencing, libraries were automatically transferred to one lane of the flow-cell, where DNA binding occurred and clusters were generated. Clusters were then imaged as individual tiles in the flow cell prior to base-calling, filtering and quality scoring. For further details of sequencing using the Illumina MiSeq (see Ravi et al. 2018).

\section{Bioinformatics analysis}

Reads were aligned to the revised Cambridge reference sequence (NC_012920) using the BWA-MEM algorithm (v0.7.12) and were sorted and indexed using SAMtools (v0.1.18) (Li and Durbin 2009; Li et al. 2009). The average number of reads was 32,640 per sample with $92.5 \%$ on target and an average depth of 182.1. Variant calling was performed using the Genome Analysis Toolkit (GATK; v3.2.2) with tenploidy settings that assume ten copies of the mitochondrial genome (McKenna et al. 2010). Variant calling results were input into HaploGrep2 (v2.1.0) for determination of mtDNA haplogroup (Weissensteiner et al. 2016). HaploGrep2 used PhyloTree 17 as a reference and provided a quality score for each result dependent on the reliability of the haplogroup assignment.

\section{Blood collection and platelet isolation}

Of the 383 volunteers whose mtDNA was successfully sequenced, 30 volunteers from haplogroups $H, J, T$ and $U$ were recalled to donate fresh blood. Volunteers were ranked according to HaploGrep2 quality score, so that the volunteer samples with the highest haplogroup calling quality score could be selected for recall where possible. Whole blood $(20 \mathrm{~mL})$ was collected from each healthy volunteer in ethylenediaminetetraacetic acid (EDTA) vacutainers (BD Biosciences, USA). A tourniquet was used to assist blood collection only when absolutely required and in each case was used for $<30 \mathrm{~s}$ to minimise the risk of platelet activation. Within $30 \mathrm{~min}$ of collection, blood was layered on top of Optiprep ${ }^{\mathrm{TM}}$ density gradient medium $(1.063 \mathrm{~g} / \mathrm{mL})$ using a Pasteur pipette before centrifugation $(350 \mathrm{~g}, 15 \mathrm{~min}$, no brake). Following this, the top $3 / 4$ of platelet-rich plasma (PRP) was removed and the remaining $1 / 4$ (likely to be contaminated with red blood cells) was discarded. Prostaglandin $\mathrm{I}_{2}\left(\mathrm{PGI}_{2}\right)$ was added to the PRP to a final concentration of $1 \mu \mathrm{g} / \mathrm{mL}$ to prevent platelet activation. The PRP was then centrifuged ( $1500 \mathrm{~g}, 15 \mathrm{~min}$, no brake) to generate a platelet pellet. The platelet pellet was subsequently resuspended in $\mathrm{Ca}^{2+}$-free PBS (Life technologies, Paisley, UK) supplemented with $\mathrm{PGI}_{2}(1 \mu \mathrm{g} / \mathrm{mL})$ before centrifugation $(1500 \mathrm{~g}$, 15 min, no brake).

\section{Platelet quantification}

The absorbance of the platelet suspension, diluted tenfold, was measured $(750 \mathrm{~nm})$. Absorbance data were input into the following equation to quantify platelets, where $\mathrm{k}$ is a 
geometrical factor equal to 1.33 for a flat bottom 96-well microplate, $y$ is the wavelength used $(750 \mathrm{~nm}), \mathrm{R}$ is the dilution factor of the sample (Walkowiak et al. 1997).

$$
\left(\frac{6.23}{2.016-\left(\mathrm{k} \times \mathrm{y} \times\left(\frac{\text { reading-blank }}{800}\right)\right.}-3.09\right) \times R=\text { platelets } \times 10^{8} / \mathrm{mL}
$$

\section{Platelet extracellular flux analysis}

\section{Preparation}

Following quantification, platelets were seeded in $\mathrm{Ca}^{2+}$-free PBS in two CellTak (Corning ${ }^{\mathrm{TM}}$ )-coated XF 96-well cell culture microplates, one for a mitochondrial stress test and one for a respiratory complex assay $\left(1 \times 10^{7}\right.$ platelets $/ 50 \mu \mathrm{L} /$ well). Plates were then centrifuged ( $200 \mathrm{~g}$, 1 min, no brake) to ensure platelet adherence.

\section{Mitochondrial stress test}

The PBS in one of the 96-well microplates containing platelets was replaced with unbuffered Seahorse XF base medium ( $175 \mu \mathrm{L} /$ well $)$ supplemented with glucose $(25 \mathrm{mM}), \mathrm{L}$-glutamine $(2 \mathrm{mM})$, sodium pyruvate $(1 \mathrm{mM})$, pre-warmed to $37^{\circ} \mathrm{C}(\mathrm{pH} 7.4)$ before incubation $\left(1 \mathrm{~h}, 37^{\circ} \mathrm{C}, 0 \% \mathrm{CO}_{2}\right)$. XF analysis was then performed as per Ball et al. 2016 using the Seahorse XFe96 Analyser. Briefly, baseline oxygen consumption rate (OCR) was measured prior to the acute injection of flutamide, 2-hydroxyflutamide or tolcapone $(30,125$ or $250 \mu \mathrm{M}$; concentrations which have previously been used to induce mitochondrial dysfunction in the absence of whole cell death (Kamalian et al. 2015; Ball et al. 2016)). Following compound injection into the platelet medium, a mitochondrial stress test was performed utilising oligomycin (ATP synthase inhibitor; $1 \mu \mathrm{M}$ ), carbonyl cyanide 4-(trifluoromethoxy) phenylhydrazone (FCCP; uncoupler; $0.5 \mu \mathrm{M}$ ) and rotenone/antimycin A (complex I/III inhibitors, respectively; $1 \mu \mathrm{M}$ each). This enabled the following parameters to be measured: basal, maximum and ATPlinked respiration, spare respiratory capacity and proton leak (see Ball et al. 2016 for derivation of parameters).

\section{Respiratory complex assays}

These assays were performed as described previously (Ball et al. 2016). Briefly, PBS was replaced with mitochondrial assay solution buffer (MAS: $\mathrm{MgCl}_{2} ; 5 \mathrm{mM}$, mannitol; $220 \mathrm{mM}$, sucrose; $70 \mathrm{mM}, \mathrm{KH}_{2} \mathrm{PO}_{4} ; 10 \mathrm{mM}$, HEPES; $2 \mathrm{mM}$,
EGTA; $1 \mathrm{mM}$, bovine serum albumin (BSA); $0.4 \% \mathrm{w} / \mathrm{v}$ ) containing constituents to permeabilise cells and stimulate oxygen consumption via complex I (ADP; $4.6 \mathrm{mM}$, malic acid; $30 \mathrm{mM}$, glutamic acid; $22 \mathrm{mM}, \mathrm{BSA} ; 30 \mu \mathrm{M}, \mathrm{PMP} ; 1 \mathrm{nM}$ ), complex II (ADP; $4.6 \mathrm{mM}$, succinic acid; $20 \mathrm{mM}$, rotenone; $1 \mu \mathrm{M}$, BSA; $30 \mu \mathrm{M}$, PMP; $1 \mathrm{nM}$ ), complex III (ADP; $4.6 \mathrm{mM}$, duroquinol; $500 \mu \mathrm{M}$, rotenone; $1 \mu \mathrm{M}$, malonic acid; $40 \mu \mathrm{M}, \mathrm{BSA} ; 0.2 \% \mathrm{w} / \mathrm{v}$, PMP; $1 \mathrm{nM}$ ) or complex IV (ADP; $4.6 \mathrm{mM}$, ascorbic acid; $20 \mathrm{mM}, \mathrm{N}, \mathrm{N}, \mathrm{N}^{\prime}, \mathrm{N}^{\prime}$-tetramethyl-pphenylenediamine (TMPD); $0.5 \mathrm{mM}$, antimycin A; $2 \mathrm{mM}$, BSA; $30 \mathrm{mM}$, PMP; $1 \mathrm{nM}$ ). Following a basal measurement period (2-hydroxyflutamide was injected (15, 30, 125 or $250 \mu \mathrm{M}$ ) followed by a mitochondrial stress test. For assessment of complex IV-driven respiration, potassium azide (complex IV inhibitor), rather than rotenone/antimycin A (complex I and III inhibitors, respectively), was injected to prevent mitochondrial respiration.

\section{Statistical analysis}

Results from the 30 healthy volunteers were pooled according to haplogroup; $H(n=8), J(n=6), T(n=7)$ and $U$ $(n=9)$. Results from mitochondrial stress tests and complex assays were assessed for statistically significant differences between haplogroups. The following parameters were selected for comparison: basal, maximum and ATP-linked respiration, spare respiratory capacity, proton leak and complex I-IV-driven respiration.

Pearson's correlation coefficient was calculated to establish if parameters of basal mitochondrial function: spare respiratory capacity, basal respiration; could be used to predict outputs of dysfunction: $\mathrm{EC}_{50}$ ATP-linked respiration (flutamide, 2-hydroxyflutamide, tolcapone) and if basal complex I/II-driven respiration could be used to predict 2-hydroxyflutamide $\mathrm{EC}_{50}$ complex I/II-driven respiration. Results from platelets of all haplogroups were pooled to provide a sufficient $n$ number for the correlation analysis. $\mathrm{EC}_{50}$ values were determined by nonlinear regression analysis using GraphPad Prism 7.0 following mean centring using SPSS v24. Normality was assessed using a Shapiro-Wilk test. Statistical significance was determined by a one-way ANOVA for parametric data followed by a Dunnett's post-hoc test using StatsDirect 2.7.9. Differences were determined to be significant at $p<0.05$.

\section{Results}

\section{Phylogenetic distribution of samples}

383 healthy volunteer samples were sequenced successfully. As well as determining haplogroup classification and quality information, HaploGrep2 provided the resultant 


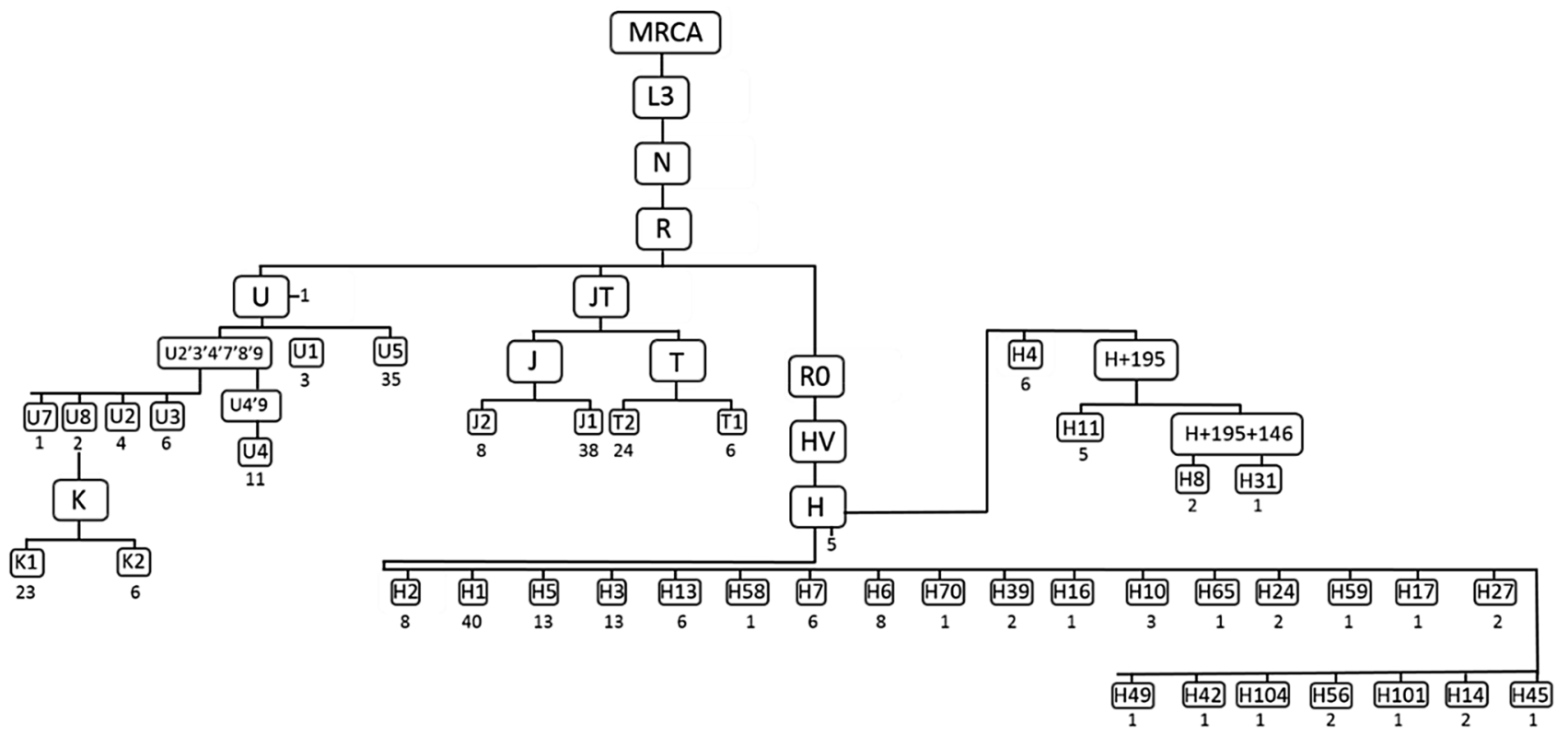

Fig. 1 Phylogenetic tree of the five most common haplogroups in the study cohort. Genotyping results from 383 healthy volunteers were classified into mitochondrial sub-haplogroups; the five most common

phylogenetic tree of all samples (Supplementary Fig. 3). A simplified version of this phylogenetic tree is displayed in Fig. 1. Haplogroup assignment and SNPs of the 30 volunteers who donated blood for subsequent platelet function studies are detailed in Supplementary Fig. 4.

\section{Basal mitochondrial respiratory function}

Assessment of basal mitochondrial respiratory function using a mitochondrial stress test identified increased respiration in haplogroup $H$ and $U$ platelets compared with haplogroups $J$ and $T$, though this did not reach statistical significance (Fig. 2a). When each parameter was considered as a proportion of maximal respiration, there was no significant difference in ATP-linked respiration, spare respiratory capacity and proton leak between haplogroups (Fig. 2b-e).

In permeabilised platelets, complex I-driven respiration was significantly lower in haplogroup $J$ platelets (Fig. 3a), whereas haplogroup $U$ platelets exhibited significantly increased complex I- and III-driven respiration compared with the remaining haplogroups (Fig. 3a, c). Haplogroup $U$ platelets also had the greatest complex IV-driven respiration (Fig. 3d). Complex II-driven respiration was similar across platelets of all haplogroups (Fig. 3b). haplogroups are displayed here. The number of samples is displayed under each group. MRCA most recent common ancestor

\section{Flutamide-, 2-hydroxyflutamide- and tolcapone-treated mitochondrial respiration}

Multiple parameters of mitochondrial function were measured following acute treatment of platelets with $250 \mu \mathrm{M}$ flutamide (Fig. 4), 2-hydroxyflutamide (Fig. 5) or tolcapone (Fig. 6). No significant differences between haplogroups were identified and generally, inter-haplogroup variation upon treatment with $250 \mu \mathrm{M}$ of each compound was inline with inter-haplogroup variation with control treatment. Notably, haplogroup $T$ platelets demonstrated the greatest increase in proton leak when treated with $250 \mu \mathrm{M}$ flutamide or 2-hydroxyflutamide. For the results of mitochondrial stress tests using 10,30 and $125 \mu \mathrm{M}$ compound concentrations see Supplementary Figs. 6, 7, 8.

\section{2-hydroxyflutamide-induced respiratory complex I and II dysfunction}

Haplogroup $J$ platelets had significantly lower complex I-driven respiration both when untreated and when treated with $250 \mu \mathrm{M} 2$-hydroxyflutamide. This was also reflected in the $\mathrm{EC}_{50}$ for complex I-driven respiration in haplogroup $J$ platelets, which was significantly less than the remaining haplogroups (Table 1). Haplogroup $U$ platelets had consistently greater complex I-driven respiration, which reached significance at $125 \mu \mathrm{M}$ (Fig. 7a).

Complex II-driven respiration was similar in all haplogroups, except upon treatment with $125 \mu \mathrm{M}$ 


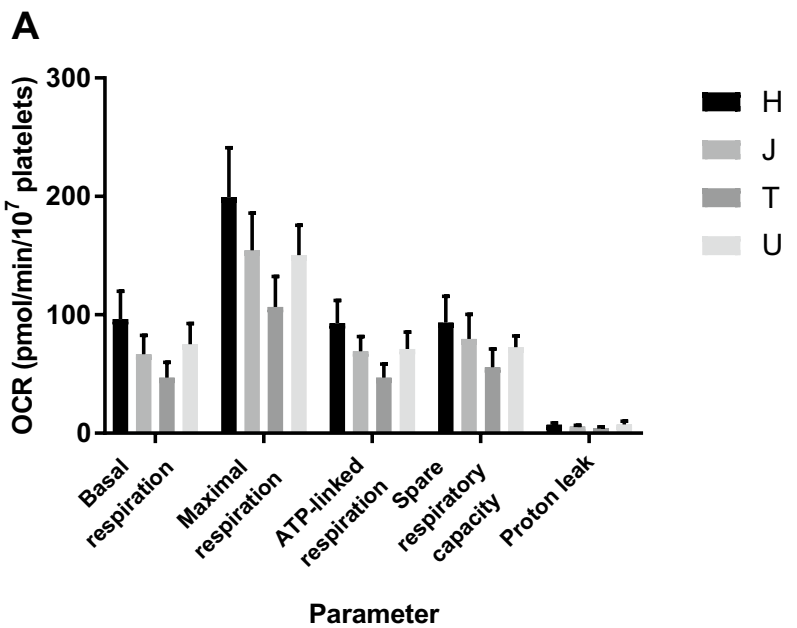

B

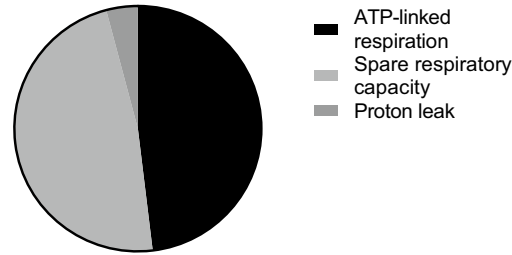

C

Haplogroup H

D

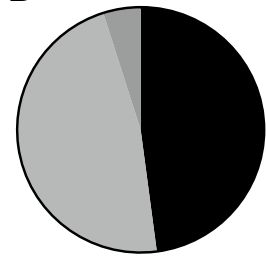

Haplogroup T

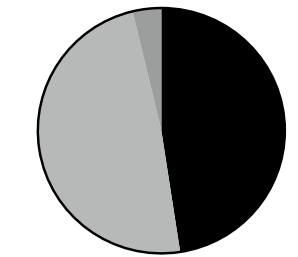

Haplogroup J

E

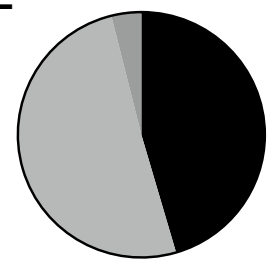

Haplogroup U

Fig. 2 Basal mitochondrial function. Extracellular flux analysis was used to perform a mitochondrial stress test using platelets from donors of haplogroups $H, J, T$ and $U$. a basal, maximal and ATPlinked respiration, spare respiratory capacity and proton leak of platelets from each haplogroup. b-e: ATP-linked respiration, spare respiratory capacity and proton leak as a proportion of maximal respiration. There were no statistically significant differences between haplogroups. Data are presented as mean + SEM of $n \geq 6$ independent experiments. $O C R$ oxygen consumption rate

2-hydroxyflutamide, where haplogroup $U$ had a higher activity. When treated with $250 \mu \mathrm{M}$ 2-hydroxyflutamide, all haplogroups demonstrated a similar reduction in activity (Fig. 7b).

\section{Correlation between basal and treated mitochondrial respiratory function}

The ability of basal values of mitochondrial function to predict responses to compound treatment was determined by derivation of the correlation coefficient between basal and treated outputs of mitochondrial function (Table 2). The spare respiratory capacity of untreated platelets provided the strongest positive correlation $(R$ value nearest to +1$)$ with the $\mathrm{EC}_{50}$ ATP-linked respiration of flutamide and 2-hydroxyflutamide, but not tolcapone. Basal respiration and complex I/ II-driven respiration, however, failed to strongly correlate $(R$ value near 0$)$ with the $\mathrm{EC}_{50}$ of any of the test compounds.

\section{Discussion}

The abundance of mtDNA and absence of nuclear DNA in platelets makes these cell fragments an attractive model to study the effect of mtDNA variation upon mitochondrial function. In this study, the mitochondrial haplogroup of 383 healthy volunteers was determined using whole mitochondrial genome sequencing. This information was used to select individuals from the most common mitochondrial haplogroups in England; $H, J, T$ and $U$; whom were recalled to donate platelets. This enabled mitochondrial function differences between haplogroups to be assessed in platelets at basal state and in platelets treated with therapeutic compounds known to induce mitochondrial dysfunction; flutamide, 2-hydroxyflutamide (primary metabolite of flutamide) and tolcapone.

Total mitochondrial respiration did not vary significantly between haplogroups, though haplogroup $J$ and $T$ platelets had lower respiration compared with haplogroups $H$ and $U$ across multiple treatment doses. Treatment of permeabilised platelets provided with respiratory complex-specific substrates enabled inter-haplogroup differences to be assessed at the sub-mitochondrial level. Here, haplogroup $J$ platelets had significantly lower complex I-driven respiration both when untreated and when treated with $250 \mu \mathrm{M}$ 2-hydroxyflutamide. This was also reflected in the $\mathrm{EC}_{50}$ complex I-driven respiration of 2-hydroxyflutamide in haplogroup $J$ platelets, which was significantly less than the remaining haplogroups. The reduced complex I-driven respiration of haplogroup $J$ platelets could be associated with the non-synonymous SNPs characteristic of this haplogroup including A10398G (rs2853826) and G13708A (rs28359178) in regions encoding complex I subunits, MT-ND3 and MT-ND5, respectively (Czarnecka et al. 2010; Pignataro et al. 2017). Work utilising 
A
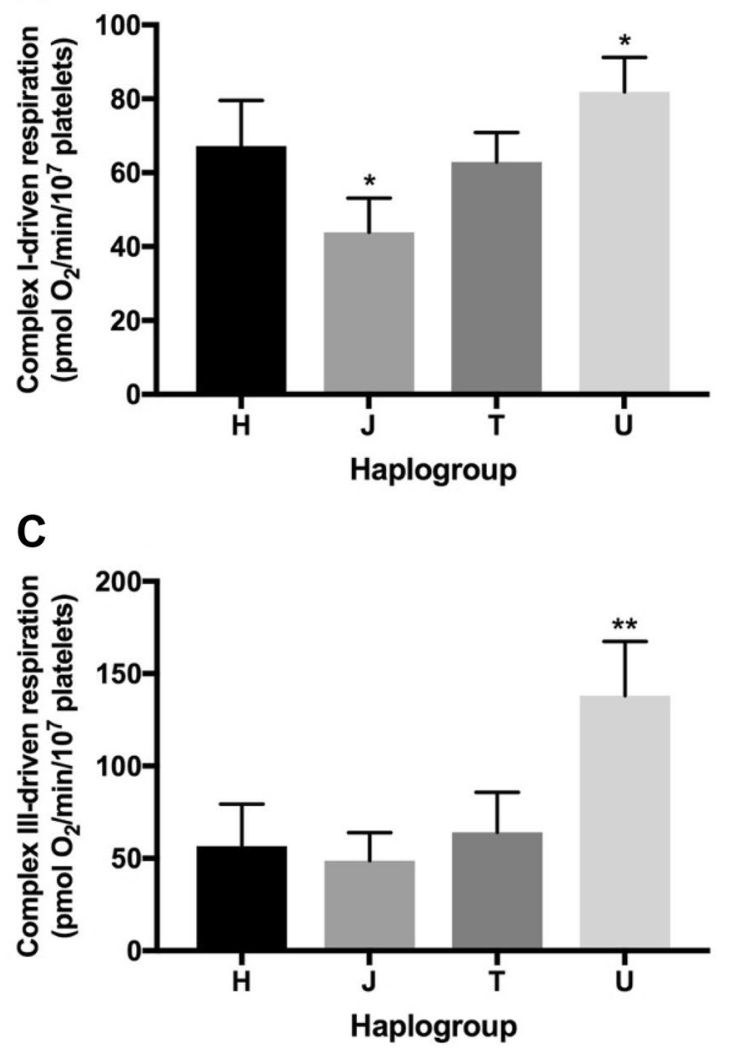

Fig. 3 Basal complex-driven respiration. Extracellular flux analysis of platelets from donors of haplogroups $H, J, T$ and $U$ was performed using uncoupled, permeabilised platelets, with respiration driven via the addition of substrates specific to complexes I (a), II (b), III (c) or IV (d). Complex-driven respiration was defined by the maximal res-

transmitochondrial cybrids has noted that these mutations result in a significant difference in ATP-production driven specifically via complex I/II, between haplogroups $J$ and $U$, which mirrors the current findings (Ghelli et al. 2009). Three of the haplogroup $J$ donor samples used in this study (9, 16 and 30; see Supplementary Fig. 4) also contained the T3394C SNP within the region encoding MT-ND1 (complex I), characteristic of the J1c1 sub-haplogroup (Liang et al. 2009).

The fact that significant differences in total mitochondrial respiration between haplogroups were not observed may be a product of the limited size of this cohort and the heterogeneity encompassed within haplogroups. However, it should be noted that work by Strobbe et al. using transmitochondrial cybrids of haplogroups $H, J, T, U$ and $K$ has also reported little variance in basal bioenergetic function but significant differences at the respiratory complex level
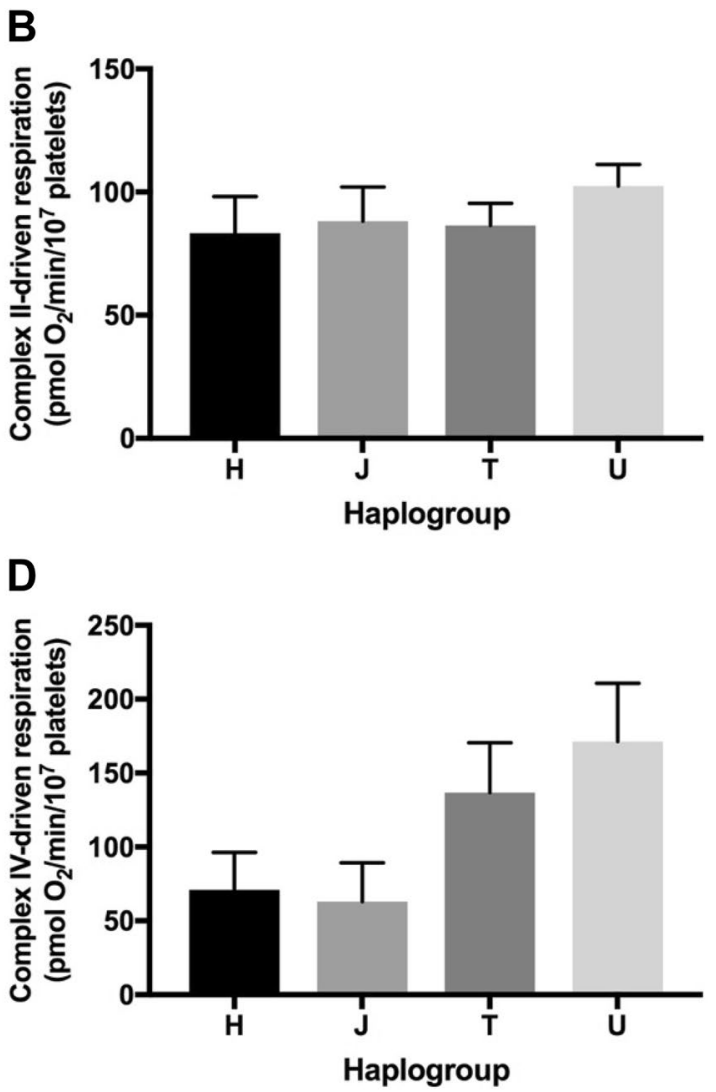

piration when treated with substrates specific to the complex of interest. Statistical significance compared with other haplogroups, e.g., $\mathrm{H}$ vs. non-H: $* p<0.05, * * p<0.01, * * * p<0.001$. Data are presented as mean \pm SEM of $n \geq 6$ independent experiments

when using rotenone as a complex-I specific inhibitor. Specifically, haplogroup $J$ was found to be more sensitive, in agreement with the overall findings of the current study (Strobbe et al. 2018).

In contrast to complex I, complex II is entirely encoded in the nuclear genome; therefore, a difference in complex II activity between haplogroups would not be anticipated; indeed this was the case in this work, with no significant difference in platelet complex II-driven respiration between haplogroups (Chinnery and Hudson 2013). Overall, the concurrence in the findings presented in this manuscript with other studies in the field provides additional confidence in the utility of platelets as an ex vivo model for studying the effect of mitochondrial genotype on susceptibility to druginduced mitochondrial dysfunction.

This study also pooled the results from all haplogroups to investigate if the extent of drug-induced mitochondrial 
A

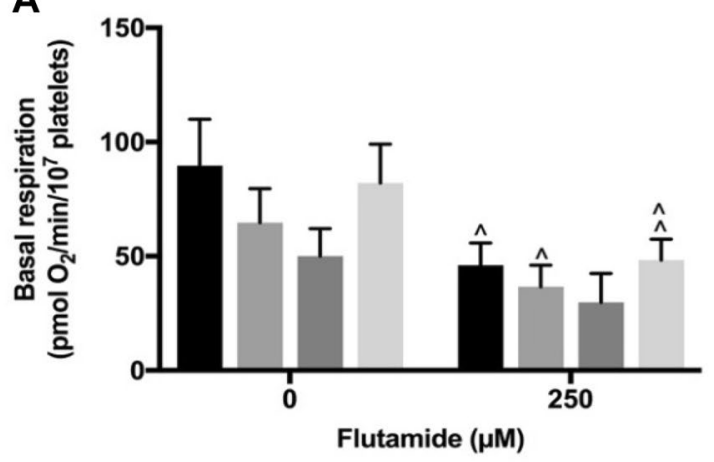

C

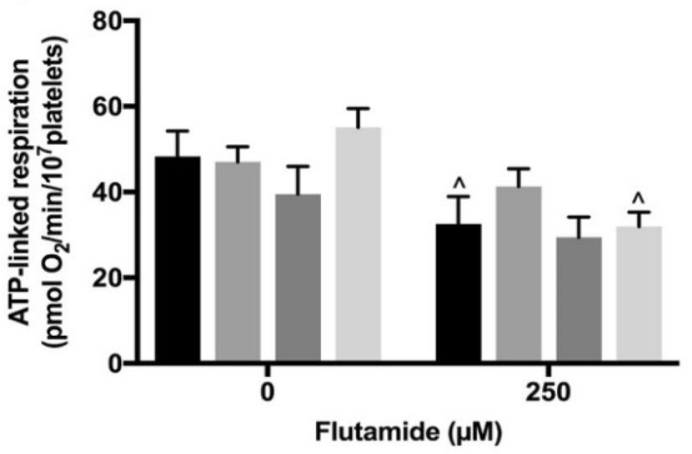

E

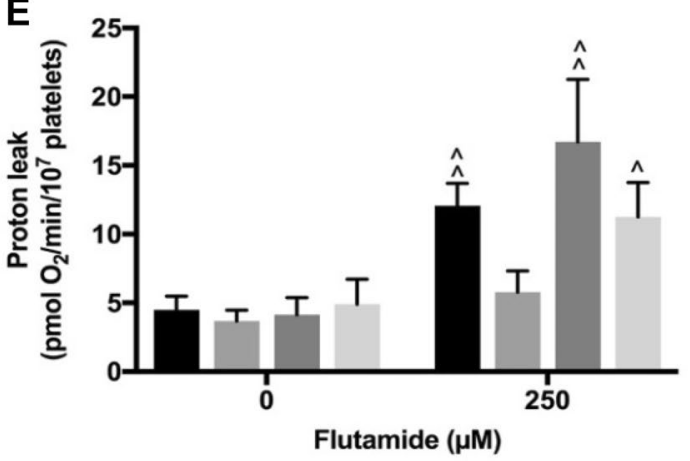

Fig. 4 Mitochondrial respiratory function of flutamide-treated platelets. Extracellular flux analysis of platelets from donors of haplogroups $H, J, T$ and $U$ was performed following acute treatment with flutamide. Changes in basal respiration (a), maximal respiration (b), ATP-linked respiration (c), spare respiratory capacity (d) and proton leak (e) were measured. Statistical significance vs. vehicle control:

dysfunction could be predicted from the assessment of mitochondrial function at basal state (untreated). Spare respiratory capacity was not significantly different between haplogroups in untreated platelets, but was significantly
B

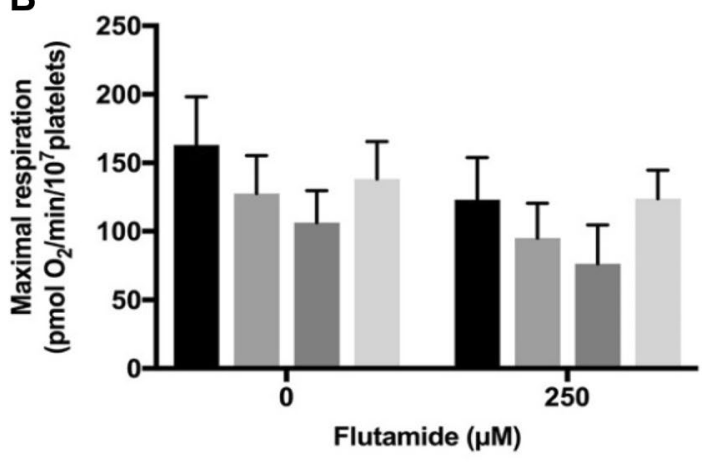

D
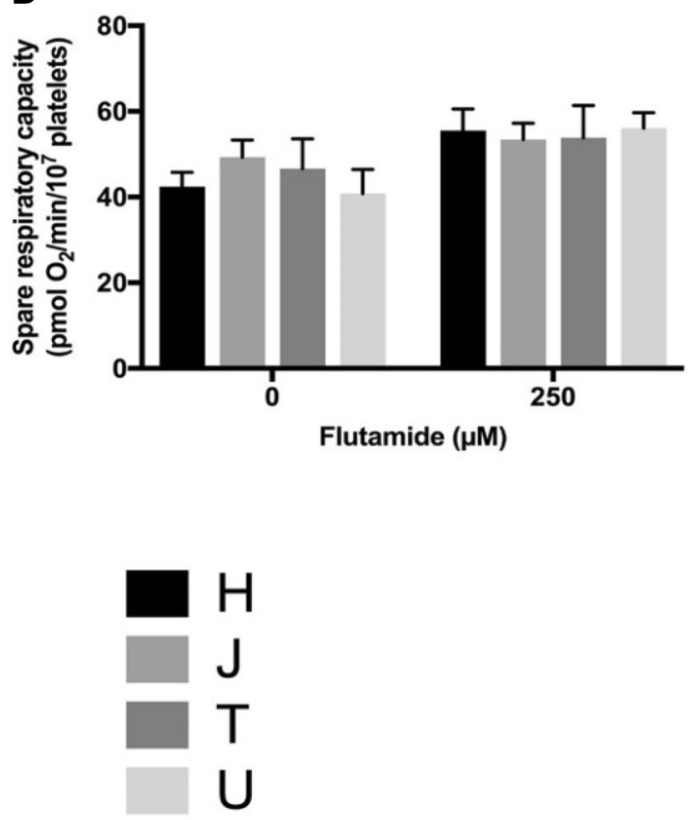

${ }^{\wedge} p<0.05,{ }^{\wedge} \wedge p<0.01,{ }^{\wedge} \wedge p<0.001$. There were no statistically significant differences between haplogroups. For clarity, only data from $250 \mu \mathrm{M}$ treatment are shown (see Supplementary Fig. 6 for full concentration range) Data are presented as mean + SEM of $n \geq 6$ independent experiments

positively correlated with the $\mathrm{EC}_{50}$ of ATP-linked respiration in flutamide and 2-hydroxyflutamide-treated platelets, i.e., platelets with increased spare respiratory capacity were observed to have greater resistance to the 
A

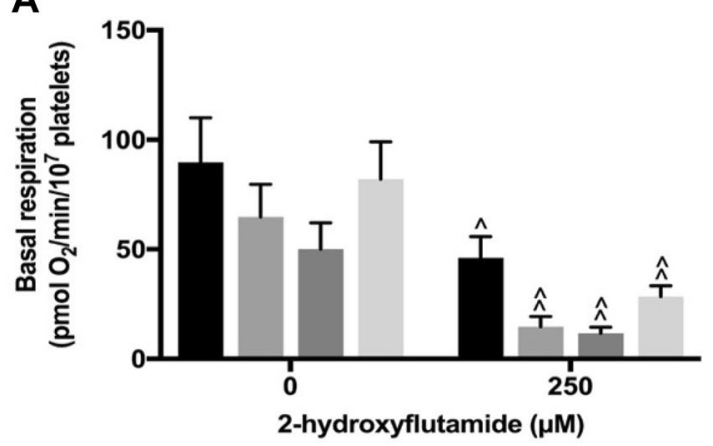

C

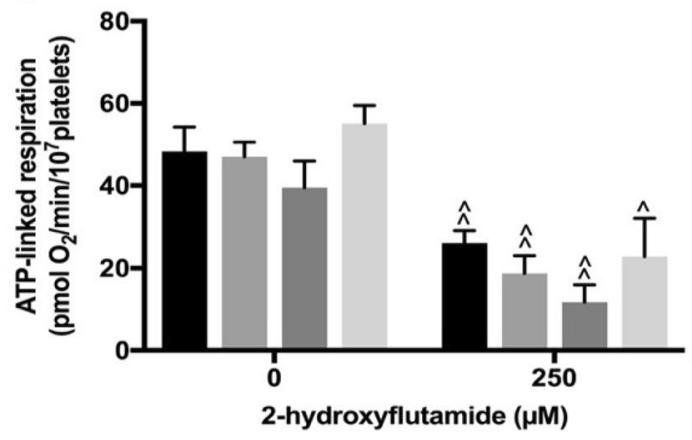

E

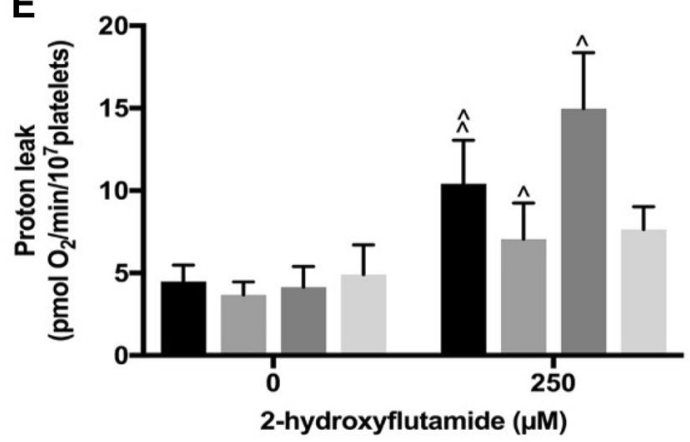

B

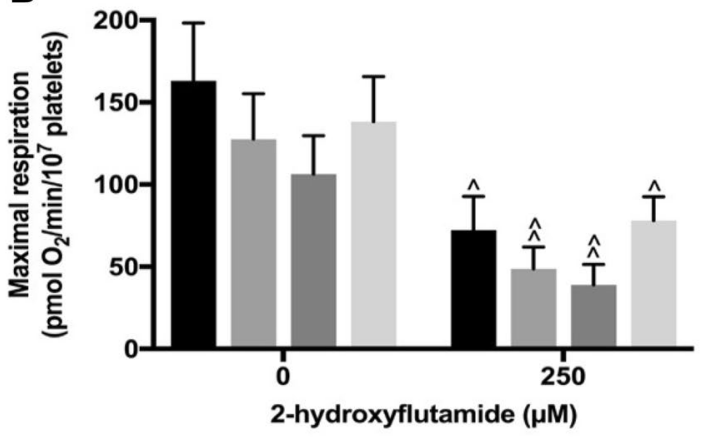

D
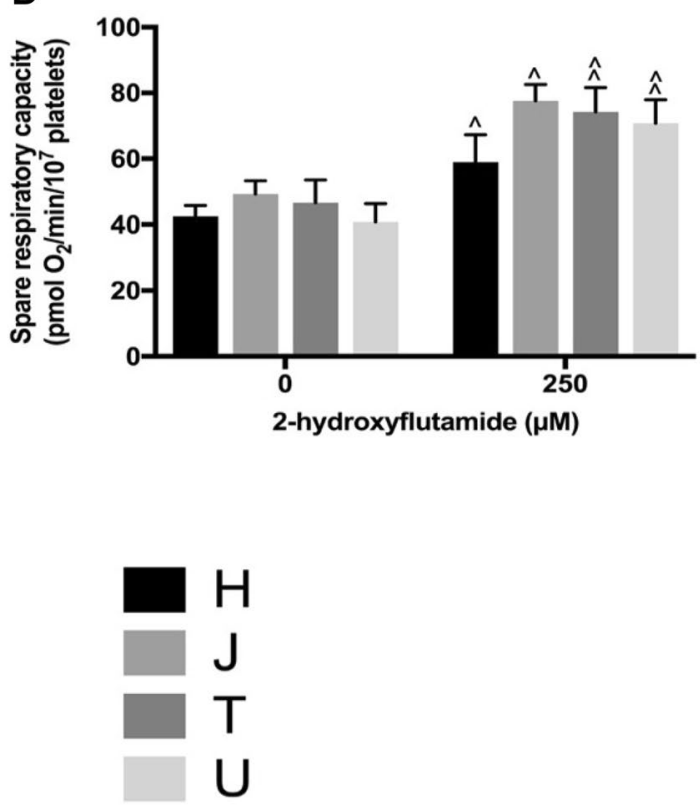

Fig. 5 Mitochondrial respiratory function of 2-hydroxyflutamidetreated platelets. Extracellular flux analysis of platelets from donors of haplogroups $H, J, T$ and $U$ was performed following acute treatment with 2-hydroxyflutamide. Changes in basal respiration (a), maximal respiration (b), ATP-linked respiration (c), spare respiratory capacity (d) and proton leak (e) were measured. Statistical signifi-

decrease in ATP-linked respiration induced by flutamide and 2-hydroxyflutamide. This observation could be due to spare respiratory capacity which is able to mitigate against 2-hydroxyflutamide-induced ETC disruption which results cance vs. vehicle control: ${ }^{\wedge} p<0.05,{ }^{\wedge} \wedge p<0.01,{ }^{\wedge \wedge} p p<0.001$. There were no statistically significant differences between haplogroups. For clarity, only data from $250 \mu \mathrm{M}$ treatment are shown (see Supplementary Fig. 7 for full concentration range). Data are presented as mean + SEM of $n \geq 6$ independent experiments

in the higher $\mathrm{EC}_{50}$. This constitutes an important finding as it demonstrates the potential utility of platelets for the prediction of drug-induced mitochondrial dysfunction based on basal state measurements. To reach the $\mathrm{EC}_{50}$ and 
A

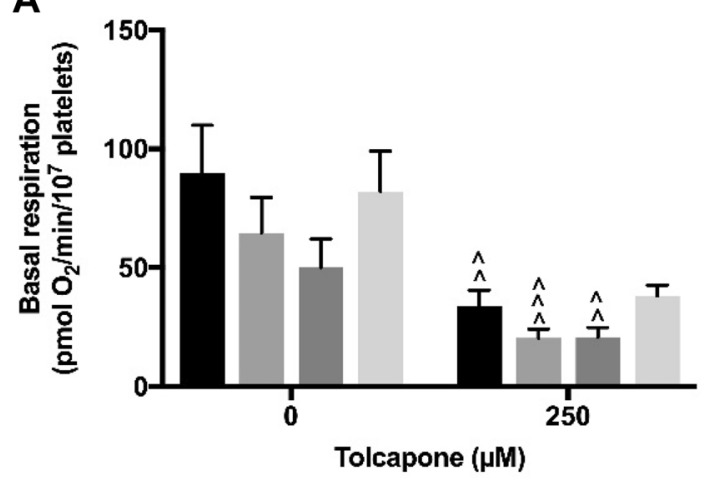

C
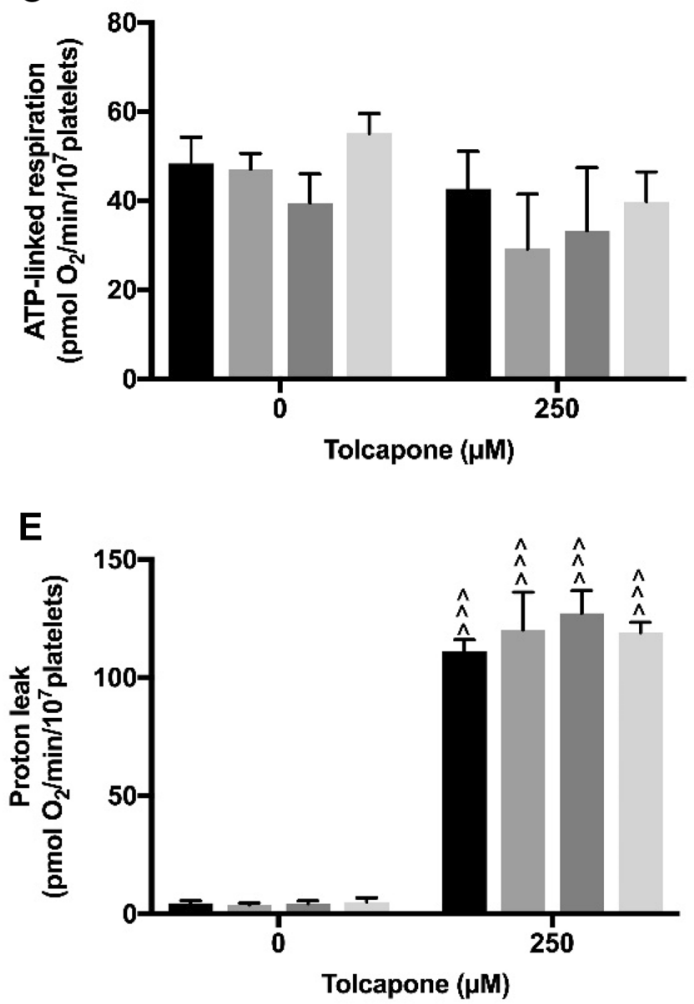

Fig. 6 Mitochondrial respiratory function of tolcapone-treated platelets. Extracellular flux analysis of platelets from donors of haplogroups $H, J, T$ and $U$ was performed following acute treatment with tolcapone. Changes in basal respiration (a), maximal respiration (b), ATP-linked respiration (c), spare respiratory capacity (d) and proton leak (e) were measured. It should be noted that there is a substantial
B

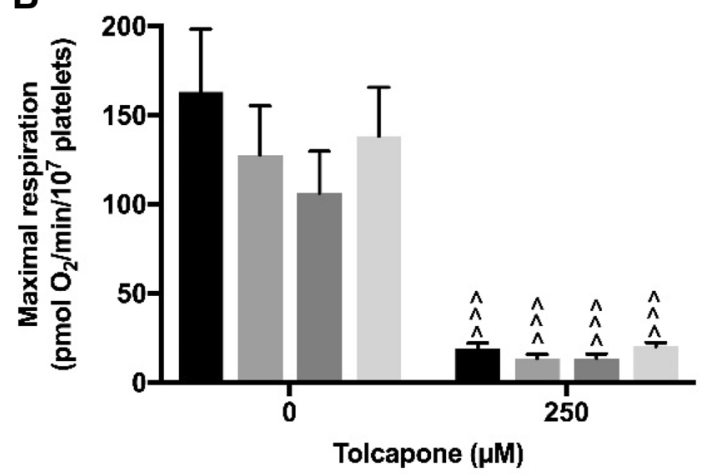

D
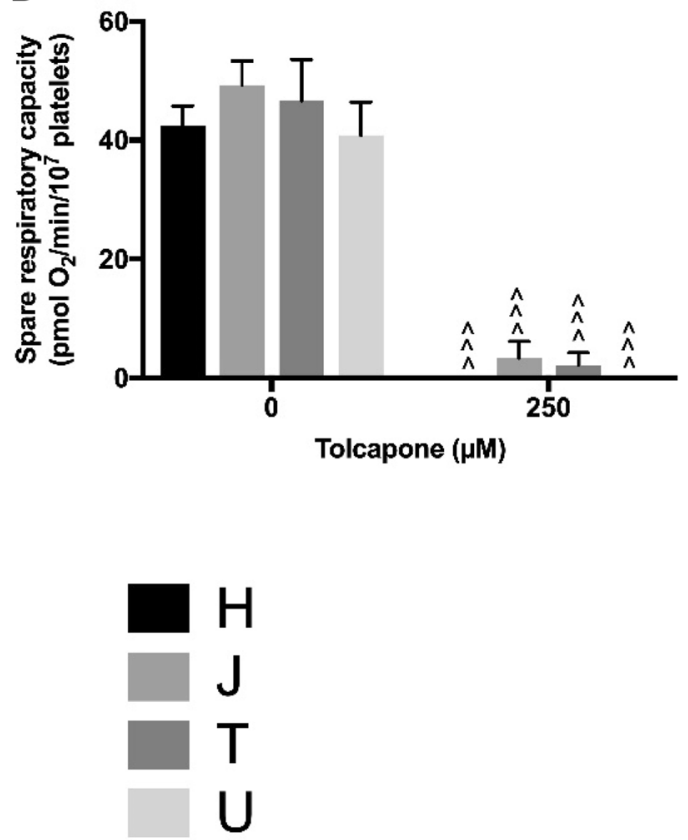

difference in the scale of the y axis between Fig. 6e and Figs. 4e and 5e. Statistical significance vs. vehicle control: ${ }^{\wedge} p<0.05,{ }^{\wedge} p<0.01$, $\wedge^{\wedge \wedge} p<0.001$. There were no statistically significant differences between haplogroups. For clarity only data from $250 \mu \mathrm{M}$ treatment are shown (see Supplementary Fig. 8 for full concentration range). Data are presented as mean + SEM of $n \geq 6$ independent experiments
Table $1 \quad \mathrm{EC}_{50}$ complex I and II-driven respiration of 2-hydroxyflutamide in platelets from donors of haplogroups $H$, $J, T$ and $U$

\begin{tabular}{lllll}
\hline Complex & \multicolumn{4}{l}{$\mathrm{EC}_{50}$ complex-driven respiration $(\mu \mathrm{M})$} \\
\cline { 2 - 5 } & $H$ & $J$ & $T$ & $U$ \\
\hline I & $118 \pm 16.2$ & $49.3 \pm 5.55(0.0390)$ & $69.0 \pm 9.72$ & $70.8 \pm 9.47$ \\
II & $144 \pm 12.1$ & $63.1 \pm 3.92(0.0200)$ & $79.0 \pm 8.96$ & $110 \pm 8.80$ \\
\hline
\end{tabular}

$\mathrm{EC}_{50}$ complex-driven respiration refers to the concentration of 2-hydroxyflutamide required to reduce complex I/II-driven respiration by $50 \%$. Data are presented as mean \pm SEM ( $p$ value) of $n \geq 6$ independent experiments. $P$ value indicates statistical significance compared with other haplogroups and is only shown if $<0.05$. 


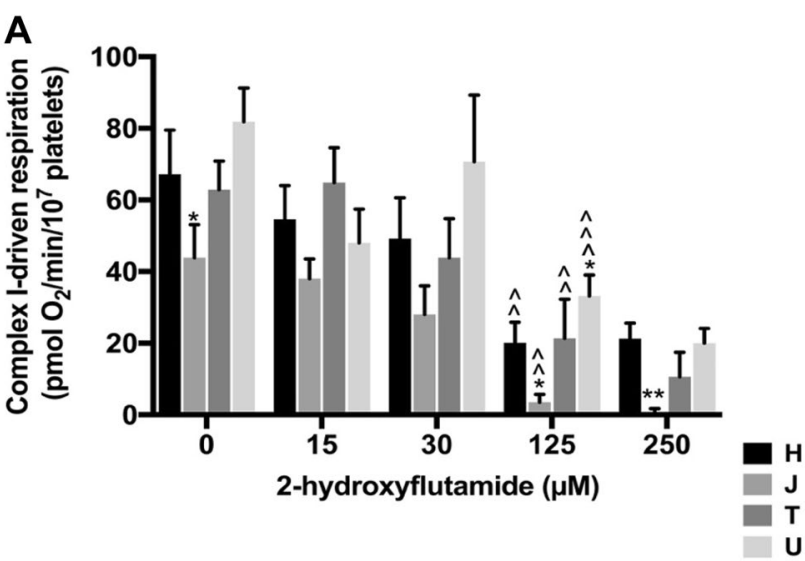

B

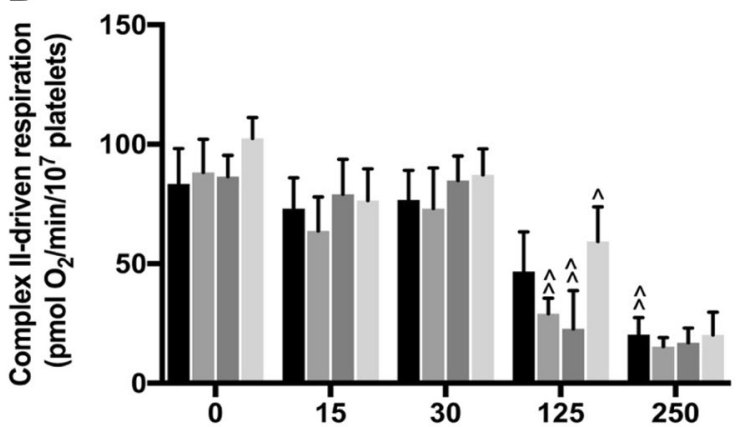

Fig. 7 Complex I and II-driven respiration in 2-hydroxyflutamidetreated platelets. Extracellular flux analysis of permeabilised platelets from donors of haplogroups $H, J, T$ and $U$ was performed in solution containing substrates specific to complexes I (a), II (b) following acute treatment with 2-hydroxyflutamide. Statistical significance vs. vehicle control: ${ }^{\wedge} p<0.05,{ }^{\wedge} p<<0.01,{ }^{\wedge} \wedge \wedge<<0.001$. For clarity only the first point of significance are shown. Statistical significance compared with other haplogroups, e.g., H vs. non-H: ${ }^{*} p<0.05$, $* * p<0.01, * * * p<0.001$. Data are presented as mean + SEM of $n \geq 6$ independent experiments observe these correlations, the concentrations used greatly exceeded the $\mathrm{C}_{\max }$ of the compounds (flutamide; $72.2 \mathrm{nM}$, 2-hydroxyflutamide; $4.4 \mu \mathrm{M}$, tolcapone; $16.5 \mu \mathrm{M}$ ); however, these concentrations have previously been used to induce mitochondrial dysfunction in the absence of whole cell death (Kamalian et al. 2015; Ball et al. 2016).

Previous research has questioned the suitability of platelets for such work due to a reported lack of spare respiratory capacity, indicating that cells are functioning at their near maximal energetic capacity under basal conditions (Chacko et al. 2013). However, in the current study, platelets were demonstrated to be a practical, primary cell model, which is amenable to the study of drug-induced mitochondrial dysfunction. Specifically, the results reported here, where platelets were typically analysed $2.5 \mathrm{~h}$ after blood samples were taken, demonstrate that freshly-isolated platelets retain mitochondrial function post their isolation from blood samples and possess significant spare respiratory capacity. This study has also demonstrated the reproducibility of bioenergetic assessments in platelet models by measuring mitochondrial respiration in platelets from two volunteers (of unknown haplogroup) across a period of 5 days (blood collected
Table 2 Correlation of basal mitochondrial function with flutamide-, 2-hydroxyflutamideand tolcapone-treated mitochondrial function

\begin{tabular}{|c|c|c|c|}
\hline Variable 1 & Variable 2 & $R$ & $P$ value \\
\hline Complex I-driven respiration & 2-Hydroxyflutamide $\mathrm{EC}_{50}$ complex I-driven respiration & 0.23 & 0.3356 \\
\hline Complex II-driven respiration & 2-Hydroxyflutamide $\mathrm{EC}_{50}$ complex II-driven respiration & 0.33 & 0.4010 \\
\hline Spare respiratory capacity & Flutamide $\mathrm{EC}_{50}$ ATP-linked respiration & 0.72 & $<0.0001$ \\
\hline y capacity & 2-Hydroxyflutamide $\mathrm{EC}_{50}$ ATP-linked respiration & 0.63 & 0002 \\
\hline y capacity & Tolcapone $\mathrm{EC}_{50}$ ATP-linked respiration & 0.35 & 0602 \\
\hline Basal respiration & Flutamide $\mathrm{EC}_{50}$ ATP-linked respiration & 0.17 & 0.3622 \\
\hline Basal respiration & 2-Hydroxyflutamide $\mathrm{EC}_{50}$ ATP-linked respiration & 0.28 & 0.1278 \\
\hline Basal respiration & Tolcapone $\mathrm{EC}_{50}$ ATP-linked respiration & 0.18 & 0.3446 \\
\hline
\end{tabular}

Variable 1 functions of platelets in a basal state, Variable 2 functions of platelets in a treated state, $R$ Pearson's correlation coefficient, $P$ value statistical significance of correlation 
and platelets analysed on days 0,2 and 4). Although maximal respiration of platelets varied between each day, there were negligible changes in parameters of mitochondrial respiratory function as a proportion of maximal respiration (Supplementary Fig. 5).

To conclude, this research has indicated that platelets are a potentially valuable ex vivo model of inter-individual variation in mtDNA and susceptibility to drug-induced mitochondrial dysfunction induced by compounds associated with adverse drug reactions. Specifically, platelets from donors of haplogroup $J$ have been found to have increased susceptibility to the acute inhibition of complex I-driven respiration by 2-hydroxyflutamide. However, in many of the tests performed, variation did not reach statistical significance, a potential artefact of the heterogeneity of mitochondrial haplogroups (Supplementary Fig. 4). Nonetheless, at a time when individual susceptibility to adverse drug reactions is not fully understood, this study provides evidence that inter-individual variation in mitochondrial genotype can be a factor in determining sensitivity to mitochondrial toxicants and will serve as a foundation for future investigations.

Supplementary Information The online version contains supplementary material available at https://doi.org/10.1007/s00204-021-02988-3.

Acknowledgements This work was supported by the Centre for Drug Safety Science supported by the Medical Research Council, United Kingdom (Grant Number G0700654); and GlaxoSmithKline as part of an MRC-CASE studentship (grant number MR/L006758/1). The authors would like to thank the Royal Liverpool Research Facility for the recruitment of volunteers and sample collection.

\section{Compliance with ethical standards}

Conflict of interest The authors have no conflicts of interest to declare that are relevant to the content of this article.

Ethical approval All procedures performed in studies involving human participants were in accordance with the ethical standards of the North West of England Research Ethics Committee (Cell Archive of HLA Typed Healthy Volunteers (HLA), CRN ID 7787, IRAS ID: 15623) with the 1964 Helsinki declaration and its later amendments or comparable ethical standards.

Open Access This article is licensed under a Creative Commons Attribution 4.0 International License, which permits use, sharing, adaptation, distribution and reproduction in any medium or format, as long as you give appropriate credit to the original author(s) and the source, provide a link to the Creative Commons licence, and indicate if changes were made. The images or other third party material in this article are included in the article's Creative Commons licence, unless indicated otherwise in a credit line to the material. If material is not included in the article's Creative Commons licence and your intended use is not permitted by statutory regulation or exceeds the permitted use, you will need to obtain permission directly from the copyright holder. To view a copy of this licence, visit http://creativecommons.org/licenses/by/4.0/.

\section{References}

Alfirevic A, Gonzalez-Galarza F, Bell C et al (2012) In silico analysis of HLA associations with drug-induced liver injury: use of a HLA-genotyped DNA archive from healthy volunteers. Genome Med 4:51. https://doi.org/10.1186/gm350

Ball AL, Kamalian L, Alfirevic A et al (2016) Identification of the additional mitochondrial liabilities of 2-Hydroxyflutamide when compared with its parent compound, flutamide in HepG2 cells. Toxicol Sci. https://doi.org/10.1093/toxsci/kfw126

Chacko BK, Kramer PA, Ravi S et al (2013) Methods for defining distinct bioenergetic profiles in platelets, lymphocytes, monocytes, and neutrophils, and the oxidative burst from human blood. Lab Invest 93:690-700. https://doi.org/10.1038/labinvest.2013.53

Chen J, Yang L, Yang A et al (2007) Maternally inherited aminoglycoside-induced and nonsyndromic hearing loss is associated with the $12 \mathrm{~S}$ rRNA C1494T mutation in three Han Chinese pedigrees. Gene 401:4-11. https://doi.org/10.1016/j.gene.2007.06.009

Chinnery PF, Hudson G (2013) Mitochondrial genetics. Br Med Bull 106:135-159

Chu CW, Hwang SJ, Luo JC et al (1998) Flutamide-induced liver injury: a case report. Chin Med J 61:678-682

Czarnecka AM, Krawczyk T, Zdrozny M et al (2010) Mitochondrial NADH-dehydrogenase subunit 3 (ND3) polymorphism (A10398G) and sporadic breast cancer in Poland. Breast Cancer Res Treat 121:511-518. https://doi.org/10.1007/s10549-009-0358-5

Eupedia (2016) European MtDNA haplogroups frequency. In: http:// www.eupedia.com/europe/european_mtdna_haplogroups_frequ ency.shtml Accessed: 7 Jul 2016. http://www.eupedia.com/europ e/european_mtdna_haplogroups_frequency.shtml

Faulkner L, Gibson A, Sullivan A et al (2016) Detection of primary T cell responses to drugs and chemicals in HLA-typed volunteers: implications for the prediction of drug immunogenicity. Toxicol Sci 154:416-429. https://doi.org/10.1093/toxsci/kfw177

Ghelli A, Porcelli AM, Zanna C et al (2009) The background of mitochondrial DNA haplogroup J increases the sensitivity of Leber's hereditary optic neuropathy cells to 2,5 -hexanedione toxicity. PLoS ONE 4:e7922. https://doi.org/10.1371/journal.pone.0007922

Grünig D, Felser A, Bouitbir J, Krähenbühl S (2017) The catecholO-methyltransferase inhibitors tolcapone and entacapone uncouple and inhibit the mitochondrial respiratory chain in HepaRG cells. Toxicol In Vitro 42:337-347. https://doi.org/10.1016/j. tiv.2017.05.013

Kamalian L, Chadwick AE, Bayliss M et al (2015) The utility of HepG2 cells to identify direct mitochondrial dysfunction in the absence of cell death. Toxicol In Vitro 29:732-740

Li H, Durbin R (2009) Fast and accurate short read alignment with Burrows-Wheeler transform. Bioinformatics. https://doi.org/10.1093/ bioinformatics/btp324

Li H, Handsaker B, Wysoker A et al (2009) The sequence alignment/map format and SAMtools. Bioinformatics. https://doi. org/10.1093/bioinformatics/btp352

Liang M, Guan M, Zhao F et al (2009) Leber's hereditary optic neuropathy is associated with mitochondrial ND1 T3394C mutation. Biochem Biophys Res Commun 383:286-292. https://doi. org/10.1016/j.bbrc.2009.03.097

Longo DM, Yang Y, Watkins PB et al (2016) Elucidating differences in the hepatotoxic potential of tolcapone and entacapone with $\operatorname{DILIsym}((\mathrm{R}))$, a mechanistic model of drug-induced liver injury. CPT Pharmacometrics Syst Pharmacol 5:31-39. https://doi. org/10.1002/psp4.12053

Massart J, Borgne-Sanchez A, Fromenty B (2018) Drug-induced mitochondrial toxicity. In: Oliveira PJ (ed) Mitochondrial biology and experimental therapeutics. Springer International Publishing, Cham, pp 269-295 
McKenna A, Hanna M, Banks E et al (2010) The genome analysis toolkit: a MapReduce framework for analyzing next-generation DNA sequencing data. Genome Res. https://doi.org/10.1101/ gr. 107524.110

Olanow CW, Watkins PB (2007) Tolcapone: an efficacy and safety review. Clin Neuropharmacol 30:287-294. https://doi. org/10.1097/wnf.0b013e318038d2b6

Pacheu-Grau D, Gómez-Durán A, Iglesias E et al (2012) Mitochondrial antibiograms in personalized medicine. Hum Mol Genet 22(6):1132-1139

Penman SL, Carter AS, Chadwick AE (2020) Investigating the importance of individual mitochondrial genotype in susceptibility to drug-induced toxicity. Biochem Soc Trans 48:787-797. https:// doi.org/10.1042/BST20190233

Pignataro D, Francia S, Zanetta F et al (2017) A missense MT-ND5 mutation in differentiated Parkinson disease cytoplasmic hybrid induces ROS-dependent DNA damage response amplified by DROSHA. Sci Rep 7:9528. https://doi.org/10.1038/s41598-017-09910-x

Ravi RK, Walton K, Khosroheidari M (2018) MiSeq: a next generation sequencing platform for genomic analysis. Methods Mol Biol 1706:223-232. https://doi.org/10.1007/978-1-4939-7471-9_12

Strobbe D, Caporali L, Iommarini L et al (2018) Haplogroup J mitogenomes are the most sensitive to the pesticide rotenone: relevance for human diseases. Neurobiol Dis 114:129-139. https://doi. org/10.1016/j.nbd.2018.02.010

Taylor RW, Turnbull DM (2005) Mitochondrial DNA mutations in human disease. Nat Rev Genet 6:389-402

Thon JN, Italiano JE (2012) Platelets: production, morphology and ultrastructure. Handb Exp Pharmacol. https://doi. org/10.1007/978-3-642-29423-5_1

Walkowiak B, Kesy A, Michalec L (1997) Microplate reader-a convenient tool in studies of blood coagulation. Thromb Res 87:95-103

Weissensteiner H, Pacher D, Kloss-Brandstatter A et al (2016) HaploGrep 2: mitochondrial haplogroup classification in the era of high-throughput sequencing. Nucleic Acids Res 44:W58-63. https ://doi.org/10.1093/nar/gkw233

Will Y, Dykens J (2014) Mitochondrial toxicity assessment in industry-a decade of technology development and insight. Expert Opin Drug Metab Toxicol 10:1061-1067

Publisher's Note Springer Nature remains neutral with regard to jurisdictional claims in published maps and institutional affiliations. 